\title{
Mean-Square Exponential Synchronization of Markovian Switching Stochastic Complex Networks with Time-Varying Delays by Pinning Control
}

\author{
Jingyi Wang, ${ }^{1}$ Chen $\mathrm{Xu}{ }^{2}$ Jianwen Feng, ${ }^{2}$ \\ Man Kam Kwong, ${ }^{3}$ and Francis Austin ${ }^{3}$ \\ ${ }^{1}$ College of Information and Engineering, Shenzhen University, Shenzhen 518060, China \\ ${ }^{2}$ College of Mathematics and Computational Science, Shenzhen University, Shenzhen 518060, China \\ ${ }^{3}$ Department of Applied Mathematics, The Hong Kong Polytechnic University, Hong Kong
}

Correspondence should be addressed to Chen Xu, xuchen_szu@szu.edu.cn

and Jianwen Feng, fengjw@szu.edu.cn

Received 19 December 2011; Accepted 2 March 2012

Academic Editor: Márcia Federson

Copyright (C) 2012 Jingyi Wang et al. This is an open access article distributed under the Creative Commons Attribution License, which permits unrestricted use, distribution, and reproduction in any medium, provided the original work is properly cited.

This paper investigates the mean-square exponential synchronization of stochastic complex networks with Markovian switching and time-varying delays by using the pinning control method. The switching parameters are modeled by a continuous-time, finite-state Markov chain, and the complex network is subject to noise perturbations, Markovian switching, and internal and outer time-varying delays. Sufficient conditions for mean-square exponential synchronization are obtained by using the Lyapunov-Krasovskii functional, Itö's formula, and the linear matrix inequality (LMI), and numerical examples are given to demonstrate the validity of the theoretical results.

\section{Introduction}

A complex network is a structure that is made up of a large set of nodes (also called vertices) that are interconnected to varying extents by a set of links (also called edges). Coupled biological systems such as neural networks and socially interacting animal species are simple examples of complex networks and so too is the Internet and the World Wide Web [1]. Complex networks, indeed, are so ubiquitously found in nature and in the modern world that it is absolutely essential for us to have a thorough understanding of their dynamical behavior, and complex networks synchronization holds particular promise for applications to many fields (e.g., population dynamics, power systems and automatic control [2-6]). 
Chaos synchronization is a phenomenon that has been widely investigated since it was first discovered by Pecora and Carroll in 1990, and it is a process in which two or more dynamical systems seek to adjust a certain prescribed property of their motion to a common behavior in the limit as time tends to infinity [7]. Many synchronization patterns have been explored (e.g., complete synchronization [8], cluster synchronization [9], phase synchronization [10], and partial synchronization [11]), and synchronization can be achieved by the use of adaptive control [12], feedback control [13], intermittent control [14], fuzzy control [15], impulsive control [16], or pinning control [17-20].

Stochastic perturbations and time delays are important considerations when simulating realistic complex networks because signals traveling along real physical systems are usually randomly perturbed by the environmental elements [12] and time-delayed by chaotic behavior (consider, e.g., a delayed neural network or a delayed Chua's circuit). Although some results have recently appeared on the synchronization of complex networks with coupling delays ([21-27]), most of the stochastically perturbed networks that have been investigated were one dimensional in the sense that the same noise impacted all the transmitted signals ([28-30]). Results on the more realistic vector-formed perturbations (in which different nodes are subject different types of noise disturbances) are scanty with $[12,31]$ being the only such results that have been reported on the stochastic synchronization of coupled neural networks. One popular model for stochastics in the sciences and industries is the Markovian switching model driven by continuous-time Markov chains ([32-37]), and Mao $[32,33]$ considered the stability of stochastic delayed differential equations using this model while others ([34-36]) discussed the exponential stability of stochastic delayed neural networks. Liu et al. [37], on the other hand, investigated the synchronization of discrete-time stochastic complex networks with Markovian jumping and mode-dependent mixed time delays.

Pinning control is a technique that applies controllers to only a small fraction of the nodes in a network, and the technique is important because it greatly reduces the number of controlled nodes for real-world complex networks (which, in most cases, is huge). In fact, pinning control can be so effective for some networks that a single pinning controller is required for synchronization, namely, for complex networks that have either a symmetric or an asymmetric coupling matrix (Chen et al. [17]). Other pinning schemes, on the other hand, are capable of globally and exponentially stabilizing a network onto a homogeneous state by using an optimal combination of the number of pinned nodes and the feedback control gain (Zhao et al. [18]). The dependence of the number of pinned nodes on the coupling strength, indeed, is also known for networks with a fixed network structure (Zhou et al. [19] and Zhao et al. [20]).

In this paper, we study the mean-square exponential synchronization of stochastic time-varying delayed complex networks with Markovian switching by using the pinning control method. We consider a stochastic complex network with internal time-varying delayed couplings, Markovian switching, and Wiener processes. We prove some sufficient conditions for mean-square exponential synchronization of these networks by applying the Lyapunov-Krasovskii functional method and the linear matrix inequality (LMI).

This paper is organized as follows. In Section 2, we introduce the general model for a stochastic complex network with time-varying delayed dynamical nodes and Markovian switching coupling. We also write down some preliminary definitions and theorems that will be needed for the rest of the paper. In Section 3, we establish some exponential synchronization criteria for such complex dynamical networks, and, in Section 4, we discuss a numerical example of the theoretical results. The paper concludes in Section 5. 


\section{Preliminaries}

\subsection{Notations}

Throughout this paper, $\mathbb{R}^{n}$ shall denote the $n$-dimensional Euclidean space and $\mathbb{R}^{n \times n}$ the set of all $n \times n$ real matrices. The superscript $T$ shall denote the transpose of a matrix or a vector, $\operatorname{Tr}(\cdot)$ the trace of the corresponding matrix $A^{s}=\left(A+A^{T}\right) / 2$ and $\mathbf{1}_{n}=(1,1, \ldots, 1)^{T} \in \mathbb{R}^{n}$ and $I_{n}$ the $n$-dimensional identity matrix. For square matrices $M$, the notation $M>0$ (resp., <0) shall mean that $M$ is a positive-definite (resp., negative-definite) matrix and $\lambda_{\max }(A)$, and $\lambda_{\min }(A)$ shall denote the greatest and least eigenvalues of a symmetric matrix, respectively.

Let $\left(\Omega, \mathcal{F},\left\{\mathcal{F}_{t}\right\}_{t>0}, D\right)$ be a complete probability space with a filtration $\left\{\mathscr{F}_{t}\right\}_{t>0}$ that is right continuous with $\overline{\mathcal{F}}_{0}$ containing all the $\mathcal{D}$-null sets. $C\left([-\tau, 0] ; \mathbb{R}^{n}\right)$ shall denote the family of continuous functions $\phi$ from $[-\tau, 0]$ to $\mathbb{R}^{n}$ with the uniform norm $\|\phi\|=\sup _{-\tau \leq s \leq 0}|\phi(s)|$ and $C_{\Psi_{0}}^{2}\left([-\tau, 0] ; \mathbb{R}^{n}\right)$ the family of all $\mathcal{F}_{0}$ measurable, $C\left([-\tau, 0] ; \mathbb{R}^{n}\right)$-valued stochastic variables $\xi=\{\xi(\theta):-\tau \leq \theta \leq 0\}$ such that $\int_{-\tau}^{0} \mathbb{E}|\xi(s)|^{2} d s \leq \infty$, where $\mathbb{E}$ stands for the correspondent expectation operator with respect to the given probability measure $D$.

Consider a complex network consisting of $N$ identical nodes with nondelayed and time-varying delayed linear coupling and Markovian switching

$$
\begin{aligned}
d x_{i}(t)= & \left\{f\left(t, x_{i}(t), x_{i}(t-\tau(t))\right)+\sum_{j=1, i \neq j}^{N} a_{i j}\left(r_{a_{i j}}(t)\right) \Sigma\left(x_{j}(t)-x_{i}(t)\right)\right. \\
& \left.+\sum_{j=1, i \neq j}^{N} b_{i j}\left(r_{b_{i j}}(t)\right) \Sigma\left(x_{j}\left(t-\tau_{c}(t)\right)-x_{i}\left(t-\tau_{c}(t)\right)\right)\right\} d t \\
& +\sigma_{i}\left(t, x(t), x(t-\tau(t)), x\left(t-\tau_{c}(t)\right), r_{\sigma_{i}}(t)\right) d w_{i}(t), \quad i=1,2, \ldots, N,
\end{aligned}
$$

where $x_{i}(t)=\left(x_{i 1}(t), x_{i 2}(t), \ldots, x_{i n}(t)\right)^{T} \in \mathbb{R}^{n}$ is the state vector of the $i$ th node of the network, $f\left(t, x_{i}(t), x_{i}(t-\tau(t))\right)=\left[f_{1}\left(t, x_{i}(t), x_{i}(t-\tau(t))\right), f_{2}\left(t, x_{i}(t), x_{i}(t-\tau(t))\right), \ldots, f_{n}\left(t, x_{i}(t), x_{i}(t-\right.\right.$ $\tau(t)))]^{T}$ is a continuous vector-valued function, $\Sigma=\operatorname{diag}\left(\rho_{1}, \varrho_{2}, \ldots, \varrho_{n}\right)$ is an inner coupling of the networks that satisfies $\rho_{j}>0, j=1,2, \ldots, n$, and $r_{a_{i j}}(t), r_{b_{i j}}(t)$ and $r_{\sigma_{i}}(t)$ are the continuous-time Markov processes that describe the evolution of the modes at time $t$. Here, $A\left(r_{a}(t)\right)=\left[a_{i j}\left(r_{a_{i j}}(t)\right)\right] \in \mathbb{R}^{n \times n}$ and $B\left(r_{b}(t)\right)=\left[b_{i j}\left(r_{b_{i j}}(t)\right)\right] \in \mathbb{R}^{n \times n}$ are the outer coupling matrices of the network at time $t$ at nodes $r_{a_{i j}}(t), t-\tau_{c}(t)$ and $r_{b_{i j}}(t)$, respectively, such that $a_{i j}\left(r_{a_{i j}}(t)\right) \geq 0$ for $i \neq j, a_{i i}\left(r_{a_{i i}}(t)\right)=-\sum_{j=i, j \neq i}^{N} a_{i j}\left(r_{a_{i j}}(t)\right), b_{i j}\left(r_{b_{i j}}(t)\right) \geq 0$ for $i \neq j$, and $b_{i i}\left(r_{b_{i i}}(t)\right)=-\sum_{j=i, j \neq i}^{N} b_{i j}\left(r_{b_{i j}}(t)\right) . \tau(t)$ is the inner time-varying delay satisfying $\tau \geq \tau(t) \geq 0$ and $\tau_{c}(t)$ is the coupling time-varying delay satisfying $\tau_{c} \geq \tau_{c}(t) \geq 0$. Finally, $\sigma_{i}(t, x(t), x(t-$ $\left.\tau(t)), x\left(t-\tau_{c}(t)\right), r_{\sigma_{i}}(t)\right)=\sigma_{i}\left(t, x_{1}(t), \ldots, x_{n}(t), x_{1}(t-\tau(t)), \ldots, x_{n}(t-\tau(t)), x_{1}\left(t-\tau_{c}(t)\right), \ldots, x_{n}(t-\right.$ $\left.\left.\tau_{c}(t)\right), r_{\sigma_{i}}(t)\right) \in \mathbb{R}^{n \times n}$ and $w_{i}(t)=\left(w_{i 1}(t), w_{i 2}(t), \ldots, w_{i n}(t)\right)^{T} \in \mathbb{R}^{n}$ is a bounded vector-form Weiner process, satisfying

$$
\mathbb{E} w_{i j}(t)=0, \quad \mathbb{E} w_{i j}^{2}(t)=1, \quad \mathbb{E} w_{i j}(t) w_{i j}(s)=0 \quad(s \neq t) .
$$

In this paper, $A\left(r_{a}(t)\right)$ is assumed to be irreducible in the sense that there are no isolated nodes. 
The initial conditions associated with (2.1) are

$$
x_{i}(s)=\xi_{i}(s), \quad-\check{\tau} \leq s \leq 0, i=1,2, \ldots, N,
$$

where $\check{\tau}=\max \left\{\tau(t), \tau_{c}(t)\right\}, \xi_{i} \in C_{\Psi_{0}}^{b}\left([-\check{\tau}, 0], \mathbb{R}^{n}\right)$ with the norm $\left\|\xi_{i}\right\|^{2}=\sup _{-\check{\tau} \leq s \leq 0} \xi_{i}(s)^{T} \xi_{i}(s)$, and our objective is to control system (2.1) so that it stays in the trajectory $s(t) \in \mathbb{R}^{n}$ of the system

$$
d s(t)=f(t, s(t), s(t-\tau(t))) d t
$$

by adding pinning controllers to some of the nodes. Without loss of generality, let the first $l$ nodes be controlled. Then the network is described by

$$
\begin{aligned}
d x_{i}(t)=\{ & f\left(t, x_{i}(t), x_{i}(t-\tau(t))\right)+\sum_{j=1, i \neq j}^{N} a_{i j}\left(r_{a_{i j}}(t)\right) \Sigma\left(x_{j}(t)-x_{i}(t)\right) \\
& \left.+\sum_{j=1, i \neq j}^{N} b_{i j}\left(r_{b_{i j}}(t)\right) \Sigma\left(x_{j}\left(t-\tau_{c}(t)\right)-x_{i}\left(t-\tau_{c}(t)\right)\right)+u_{i}(t)\right\} d t \\
& +\sigma_{i}\left(t, x(t), x(t-\tau(t)), x\left(t-\tau_{c}(t)\right), r_{\sigma_{i}}(t)\right) d w_{i}(t), \quad i=1,2, \ldots, N,
\end{aligned}
$$

where $u_{i}(t)(i=1,2, \ldots, N)$ are the linear state feedback controllers that are defined by

$$
u_{i}(t)= \begin{cases}-\varepsilon_{i}\left(x_{i}(t)-s(t)\right), & i=1,2, \ldots, l \\ 0, & i=l+1, l+2, \ldots, N\end{cases}
$$

where $\varepsilon_{i}>0(i=1,2, \ldots, l)$ are the control gains, denoted by $\Xi=\operatorname{diag}\left\{\varepsilon_{1}, \varepsilon_{2}, \ldots, \varepsilon_{l}, 0, \ldots, 0\right\} \in$ $\mathbb{R}^{n \times n}$. Define $e_{i}(t)=x_{i}(t)-s(t)(i=1,2, \ldots, N)$ as the synchronization error. Then, according to the controller (2.6), the error system is

$$
\begin{aligned}
d e_{i}(t)= & \left\{f\left(t, x_{i}(t), x_{i}(t-\tau(t))\right)-f\left(t, s_{i}(t), s_{i}(t-\tau(t))\right)+\sum_{j=1, i \neq j}^{N} a_{i j}\left(r_{a_{i j}}(t)\right) \Sigma\left(e_{j}(t)-e_{i}(t)\right)\right. \\
& \left.+\sum_{j=1, i \neq j}^{N} b_{i j}\left(r_{b_{i j}}(t)\right) \Sigma\left(e_{j}\left(t-\tau_{c}(t)\right)-e_{i}\left(t-\tau_{c}(t)\right)\right)+u_{i}(t)\right\} d t \\
& +\sigma_{i}\left(t, e(t), e(t-\tau(t)), e\left(t-\tau_{c}(t)\right), r_{\sigma_{i}}(t)\right) d w_{i}(t), \quad i=1,2, \ldots, N .
\end{aligned}
$$

Remark 2.1. Since the Markov chains $r_{a_{i j}}(t), r_{b_{i j}}(t)$, and $r_{\sigma_{i}}(t)$ are independent, we have an equivalent system as follows. 
Let $r(t), t>0$ be a right-continuous Markov chain on a probability space that takes values in a finite state space $S=1,2, \ldots, M$ with a generator $\Gamma=\left[\gamma_{i j}\right] \in \mathbb{R}^{M \times M}$ given by

$$
P\{r(t+\Delta)=j \mid r(t)=i\}= \begin{cases}\gamma_{i j} \Delta+o(\Delta) & \text { if } i \neq j, \\ 1+\gamma_{i i} \Delta+o(\Delta) & \text { if } i=j,\end{cases}
$$

for some $\Delta>0$. Here $\gamma_{i j}=0$ is the transition rate from $i$ to $j$ if $i \neq j$ and $\gamma_{i i}=-\sum_{i \neq j} \gamma_{i j}$,

$$
\begin{aligned}
d e_{i}(t)= & \left\{f\left(t, x_{i}(t), x_{i}(t-\tau(t))\right)-f\left(t, s_{i}(t), s_{i}(t-\tau(t))\right)+\sum_{j=1}^{N} a_{i j}(r(t)) \Sigma e_{j}(t)\right. \\
& \left.+\sum_{j=1}^{N} b_{i j}(r(t)) \Sigma e_{j}\left(t-\tau_{c}(t)\right)+u_{i}(t)\right\} d t \\
& +\sigma_{i}\left(t, e(t), e(t-\tau(t)), e\left(t-\tau_{c}(t)\right), r(t)\right) d w_{i}(t), \quad i=1,2, \ldots, N .
\end{aligned}
$$

Definition 2.2. The complex network (2.5) is said to be exponentially synchronized in mean square if the trivial solution of system (2.9) is such that

$$
\sum_{i=1}^{N} \mathbb{E}\left\|e_{i}\left(t, t_{0}, \xi_{i}\right)\right\|^{2} \leq K e^{-\kappa t}
$$

for some $K>0$ and some $\kappa>0$ for any initial data $\xi_{i} \in \mathcal{C}_{\mp_{0}}^{b}\left([-\tau, 0] ; \mathbb{R}^{n}\right)$.

Definition 2.3 (see [12]). A continuous function $f(t, x, y):[0,+\infty] \times \mathbb{R}^{n} \times \mathbb{R}^{n} \rightarrow \mathbb{R}^{n}$ is said to belong to the function class QUAD, denoted by $f \in \operatorname{QUAD}(P, \Delta, \eta, \theta)$ for some given matrix $\Sigma=\operatorname{diag}\left\{\varrho_{1}, \varrho_{2}, \ldots, \varrho_{n}\right\}$ if there exists a positive definite diagonal matrix $P=$ $\operatorname{diag}\left\{p_{1}, p_{2}, \ldots, p_{n}\right\}$, a diagonal matrix $\Delta=\operatorname{diag}\left\{\delta_{1}, \delta_{2}, \ldots, \delta_{n}\right\}$, and a constant $\eta>0, \theta>0$ such that $f(\cdot)$ satisfies the condition

$$
(x-y)^{T} P((f(t, x, z)-f(t, y, w))-\Delta \Sigma(x-y)) \leq-\eta(x-y)^{T}(x-y)+\theta(z-w)^{T}(z-w)
$$

for all $x, y, z, w \in \mathbb{R}^{n}$.

Remark 2.4. The function class QUAD includes almost all the well-known chaotic systems with or without delays such as the Lorenz system, the Rössler system, the Chen system, the delayed Chua's circuit, the logistic delayed differential system, the delayed Hopfield neural network, and the delayed CNNs. We shall simply write

$$
\check{p}=\max \left\{p_{1}, p_{2}, \ldots, p_{n}\right\}, \quad \hat{p}=\min \left\{p_{1}, p_{2}, \ldots, p_{n}\right\}, \quad \check{\delta}=\max \left\{\delta_{1}, \delta_{2}, \ldots, \delta_{n}\right\} .
$$

The following assumptions will be used throughout this paper for establishing the synchronization conditions. 
(H1) $\tau(t)$, and $\tau_{c}(t)$ are bounded and continuously differentiable functions such that $0<$ $\tau(t) \leq \tau, \dot{\tau}(t)<\bar{\tau}<1,0<\tau_{c}(t) \leq \tau_{c}$ and $\dot{\tau}_{c}(t)<\bar{\tau}_{c}<1$. Let $\stackrel{\check{\tau}}{\bar{\tau}}=\max \left\{\bar{\tau}, \bar{\tau}_{c}\right\}$.

(H2) Let $\sigma\left(t, e(t), e(t-\tau(t)), e\left(t-\tau_{c}(t)\right), r\right)=\sigma\left(t, e_{1}(t), \ldots, e_{N}(t), e_{1}(t-\tau(t)), \ldots, e_{N}(t-\right.$ $\left.\tau(t)), e_{1}\left(t-\tau_{c}(t)\right), \ldots, e_{N}\left(t-\tau_{c}(t)\right), r\right)$. Then there exist positive definite constant matrices $Y_{i 1}^{r}, Y_{i 2}^{r}$, and $\Upsilon_{i 3}^{r}$ for $i=1,2, \ldots, N$ and $r=1,2, \ldots, M$ such that

$$
\begin{aligned}
& \operatorname{Tr}\left[\sigma_{i}\left(t, e(t), e(t-\tau(t)), e\left(t-\tau_{c}(t)\right), r\right)^{T} \sigma_{i}\left(t, e(t), e(t-\tau(t)), e\left(t-\tau_{c}(t)\right), r\right)\right] \\
& \quad \leq \sum_{j=1}^{N} e_{j}(t)^{T} \Upsilon_{i 1}^{r} e_{j}(t)+\sum_{j=1}^{N} e_{j}(t-\tau(t))^{T} \Upsilon_{i 2}^{r} e_{j}(t-\tau(t))+\sum_{j=1}^{N} e_{j}\left(t-\tau_{c}(t)\right)^{T} \Upsilon_{i 3}^{r} e_{j}\left(t-\tau_{c}(t)\right) .
\end{aligned}
$$

Lemma 2.5 (see [32, 33, the generalized Itô formula]). Consider a stochastic delayed differential equation with Markovian switching of the form

$$
d x(t)=f(t, x(t), x(t-\tau), r(t)) d t+\sigma(t, x(t), x(t-\tau), r(t)) d \omega(t)
$$

on $t \geq 0$ with initial value $x_{0}=\xi \in C_{F_{0}}^{b}\left([-\tau, 0] ; \mathbb{R}^{n}\right)$, where

$$
f: \mathbb{R}^{n} \times \mathbb{R}_{+} \times S \longrightarrow \mathbb{R}^{n}, \quad \sigma: \mathbb{R}^{n} \times \mathbb{R}_{+} \times S \longrightarrow \mathbb{R}^{n \times m}
$$

Let $C^{2,1}\left(\mathbb{R}_{+} \times \mathbb{R}^{n} ; \mathbb{R}_{+}\right)$be the family of all the nonnegative functions $V(t, x, r)$ on $\mathbb{R}_{+} \times \mathbb{R}^{n} \times S$ that are twice continuously differentiable in $x$ and once differentiable in $t$. Let $V \in C^{2,1}\left(\mathbb{R}_{+} \times \mathbb{R}^{n} \times S ; \mathbb{R}_{+}\right)$. Define an operator $\mathcal{\perp}$ from $\mathbb{R}^{n} \times \mathbb{R}_{+} \times S$ to $\mathbb{R}^{n}$ by

$$
\mathcal{L}(t, x, r)=V_{t}(t, x, r)+V_{x}(t, x, r) f(t, x, r)+\frac{1}{2} \operatorname{Tr}\left[\sigma(t, x, r)^{T} V_{x x} \sigma(t, x, r)\right]+\sum_{j=1}^{M} \gamma_{i j} V(t, x, j)
$$

where $V_{t}(t, x, r)=\partial V(t, x, r) / \partial t, V_{x}(t, x, r)=\left(\partial V(t, x, r) / \partial x_{1}, \ldots, \partial V(t, x, r) / \partial x_{n}\right)$, $V_{x x}(t, x, r)=\left(\partial^{2} V(t, x, r) / \partial x_{i} x_{j}\right)_{n \times n}$. If $V \in C^{2,1}\left(\mathbb{R}_{+} \times \mathbb{R}^{n} \times S ; \mathbb{R}_{+}\right)$, then

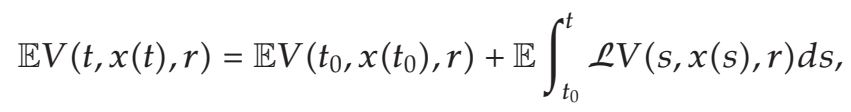

for all $\infty>t>t_{0} \geq 0$, as long as the expectations of the integrals exist. 


\section{Main Result}

Theorem 3.1. Let assumptions (H1) and (H2) be true and let $f \in \operatorname{QUAD}(P, \Delta, \eta, \theta)$. If there exist positive constants $\alpha_{r}$ and $\beta_{r}$ such that

$$
\begin{gathered}
{\left[\begin{array}{cc}
A(r)^{S}+\check{\delta} I_{N}-\Xi+\alpha_{r} I_{N} & \frac{B(r)}{2} \\
\frac{B(r)^{T}}{2} & -\beta_{r} I_{N}
\end{array}\right] \leq 0, \text { for } r=1,2, \ldots, M,} \\
\check{\tau} \leq \theta T, \quad \check{\tau} \leq(1-\theta) T, \quad 0 \leq \check{\bar{\tau}} \leq 1-\frac{\check{q}(\check{b}+\check{c})}{1+\theta}, \\
\left(\frac{1}{b_{1}+c_{1}}, \frac{1}{b_{2}+c_{2}}, \ldots, \frac{1}{b_{M}+c_{M}}\right)>\widetilde{\Gamma}^{-1} \mathbf{1}_{M},
\end{gathered}
$$

where $r>0$ is the greatest root of the equation

$$
\begin{aligned}
& \breve{q} \gamma-(1+\theta)+\frac{\check{b} \breve{q}}{1-\bar{\tau}} e^{r \tau}+\frac{\check{c} \breve{q}}{1-\bar{\tau}_{c}} e^{r \tau_{c}}=0, \\
& \widetilde{\Gamma}=\operatorname{diag}\left\{a_{1}, a_{2}, \ldots, a_{M}\right\}-\Gamma, \\
& a_{r}=\frac{\lambda_{\min }\left(2 \eta I_{n}-\check{p} \sum_{i=1}^{N} \Upsilon_{i 1}^{r}+2 \alpha_{1} P \Sigma\right)}{\check{p}}, \quad \check{a}=\max _{r \in S} a_{r}, \\
& b_{r}=\frac{\lambda_{\max }\left(\sum_{i=1}^{N} P \Upsilon_{i 2}^{r}+2 \zeta I_{N}\right)}{\hat{p}}, \quad \check{b}=\max _{r \in S} b_{r}, \\
& c_{r}=\frac{\lambda_{\max }\left(\sum_{i=1}^{N} P \Upsilon_{i 3}^{r}+2 \beta_{2} P \Sigma\right)}{\hat{p}}, \quad \check{c}=\max _{r \in S} c_{r} .
\end{aligned}
$$

Then the solutions $x_{1}(t), x_{1}(t), \ldots$ and $x_{N}(t)$ of system (2.9) are globally and exponentially stable.

Proof. By (3.3), there exists a sufficiently small constant $\theta>0$ such that

$$
\left(\frac{1}{b_{1}+c_{1}}, \frac{1}{b_{2}+c_{2}}, \ldots, \frac{1}{b_{M}+c_{M}}\right) \geq(1+\theta) \tilde{\Gamma}^{-1} \mathbf{1}
$$

Set $(1+\theta) \tilde{\Gamma}^{-1} \mathbf{1}=q=\left(q_{1}, q_{2}, \ldots, q_{M}\right)^{T}$. Then

$$
\widetilde{\Gamma} q=(1+\theta) \mathbf{1}_{M}
$$

that is,

$$
\left(b_{r}+c_{r}\right) q_{r} \leq 1, \quad a_{r} q_{r}-\sum_{s=1}^{M} \gamma_{s r} q_{r}=1+\theta
$$


For $1 \leq r \leq M$, define the Lyapunov-Krasovskii function

$$
V(t, e(t), r)=q_{r} \frac{1}{2} \sum_{i=1}^{N} e_{i}(t)^{T} P e_{i}(t)
$$

and let $\tilde{e}^{k}(t)=\left(e_{1 k}(t), e_{2 k}(t), \ldots, e_{N k}(t)\right)^{T}, k=1,2, \ldots, n$. By Lemma 2.5, we have

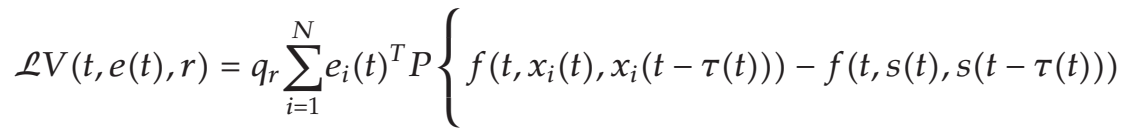

$$
\begin{aligned}
& \left.+\sum_{j=1}^{N} a_{i j}(r) \Sigma e_{j}(t)+\sum_{j=1}^{N} b_{i j}(r) \Sigma e_{j}\left(t-\tau_{c}(t)\right)+u_{i}(t)\right\} \\
& +\frac{1}{2} q_{r} \sum_{i=1}^{N} \operatorname{Tr}\left\{\sigma_{i}\left(t, x(t), x(t-\tau(t)), x\left(t-\tau_{c}(t)\right), r\right)^{T} P \sigma_{i}\right. \\
& \left.\times\left(t, x(t), x(t-\tau(t)), x\left(t-\tau_{c}(t)\right), r\right)\right\}+\sum_{s=1}^{M} \gamma_{r s} q_{s} \frac{1}{2} \sum_{i=1}^{N} e_{i}(t)^{T} P e_{i}(t) \\
& =q_{r} \sum_{i=1}^{N} e_{i}(t)^{T} P\left\{f\left(t, x_{i}(t), x_{i}(t-\tau(t))\right)-f(t, s(t), s(t-\tau(t)))-\Delta \Sigma e_{i}(t)\right\} \\
& +q_{r} \sum_{i=1}^{N} e_{i}(t)^{T} P \Delta \Sigma e_{i}(t)+q_{r} \sum_{i=1}^{N} \sum_{j=1}^{N} a_{i j}(r) e_{i}(t)^{T} P \Sigma e_{j}(t) \\
& +q_{r} \sum_{i=1}^{N} \sum_{j=1}^{N} b_{i j}(r) e_{i}(t)^{T} P \sum e_{j}\left(t-\tau_{c}(t)\right)-q_{r} \sum_{i=1}^{l} \varepsilon_{i} e_{i}(t)^{T} P \sum e_{i}(t) \\
& +\frac{1}{2} q_{r} \operatorname{Tr}\left\{\sigma_{i}\left(t, x(t), x(t-\tau(t)), x\left(t-\tau_{c}(t)\right), r\right)^{T} P \sigma_{i}\right. \\
& \left.\times\left(t, x(t), x(t-\tau(t)), x\left(t-\tau_{c}(t)\right), r\right)\right\}+\sum_{s=1}^{M} \gamma_{r s} q_{s} \frac{1}{2} \sum_{i=1}^{N} e_{i}(t)^{T} P e_{i}(t) \\
& \leq q_{r}\left\{-\eta \sum_{i=1}^{N} e_{i}(t)^{T} e_{i}(t)+\theta \sum_{i=1}^{N} e_{i}(t-\tau(t))^{T} e_{i}(t-\tau(t))+\sum_{k=1}^{n} p_{k} \varrho_{k} \delta_{k} \tilde{e}^{k}(t)^{T} \tilde{e}^{k}(t)\right. \\
& +\sum_{k=1}^{n} p_{k} \varrho_{k} \tilde{e}^{k}(t)^{T} A(r) \tilde{e}^{k}(t)+\sum_{k=1}^{n} p_{k} \rho_{k} \tilde{e}^{k}(t)^{T} B(r) \tilde{e}^{k}\left(t-\tau_{c}(t)\right) \\
& -\sum_{k=1}^{n} p_{k} \varrho_{k} \tilde{e}^{k}(t)^{T} \Xi \tilde{e}^{k}(t)
\end{aligned}
$$


Abstract and Applied Analysis

$$
\begin{aligned}
& +\frac{1}{2} \breve{p} \sum_{j=1}^{N}\left[\sum_{i=1}^{N} e_{i}(t)^{T} \Upsilon_{j 1}^{r} e_{i}(t)+\sum_{i=1}^{N} e_{i}(t-\tau(t))^{T} \Upsilon_{j 2}^{r} e_{i}(t-\tau(t))\right. \\
& \left.\left.+\sum_{i=1}^{N} e_{i}\left(t-\tau_{c}(t)\right)^{T} \Upsilon_{j 3}^{r} e_{i}\left(t-\tau_{c}(t)\right)\right]\right\}+\sum_{s=1}^{M} \gamma_{r s} q_{s} \frac{1}{2} \sum_{i=1}^{N} e_{i}(t)^{T} P e_{i}(t) \\
& =q_{r}\left\{-\eta \sum_{i=1}^{N} e_{i}(t)^{T} e_{i}(t)+\theta \sum_{i=1}^{N} e_{i}(t-\tau(t))^{T} e_{i}(t-\tau(t))+\frac{1}{2} \breve{p} \sum_{i=1}^{N} \sum_{j=1}^{N} e_{i}\left(t-\tau_{c}(t)\right)^{T}\right. \\
& \left.\times \Upsilon_{j 3}^{r} e_{i}\left(t-\tau_{c}(t)\right)\right\}+\sum_{s=1}^{M} \gamma_{r s} q_{s} \frac{1}{2} \sum_{i=1}^{N} e_{i}(t)^{T} P e_{i}(t) \\
& +q_{r}\left\{\sum_{k=1}^{n} p_{k} \varrho_{k} \tilde{e}^{k}(t)^{T} A(r) \tilde{e}^{k}(t)+\sum_{k=1}^{n} p_{k} \rho_{k} \tilde{e}^{k}(t)^{T} B(r) \tilde{e}^{k}\left(t-\tau_{c}(t)\right)\right. \\
& -\sum_{k=1}^{n} p_{k} Q_{k} \tilde{e}^{k}(t)^{T} \Xi \tilde{e}^{k}(t) \\
& +\frac{1}{2} \check{p}\left[\sum_{i=1}^{N} \sum_{j=1}^{N} e_{i}(t)^{T} \Upsilon_{j 1}^{r} e_{i}(t)+\sum_{i=1}^{N} \sum_{j=1}^{N} e_{i}(t-\tau(t))^{T} \Upsilon_{j 2}^{r} e_{i}(t-\tau(t))\right] \\
& \left.+\sum_{k=1}^{n} p_{k} \varrho_{k} \delta_{k} \tilde{e}^{k}(t)^{T} \tilde{e}^{k}(t)\right\} \\
& =q_{r}\left\{\sum_{i=1}^{N} e_{i}(t)^{T}\left(-\eta I_{N}+\frac{1}{2} \check{p} \sum_{j=1}^{N} \Upsilon_{j 1}^{r}-\alpha_{r} P \Sigma\right) e_{i}(t)+\sum_{i=1}^{N} e_{i}(t-\tau(t))^{T}\right. \\
& \times\left(\theta+\frac{1}{2} \check{p} \sum_{j=1}^{N} \Upsilon_{j 2}^{r}\right) e_{i}(t-\tau(t))+\sum_{i=1}^{N} e_{i}\left(t-\tau_{c}(t)\right)^{T}\left(\frac{1}{2} \check{p} \sum_{j=1}^{N} \Upsilon_{j 3}^{r}+\beta_{r} P \Sigma\right) \\
& \left.\times e_{i}\left(t-\tau_{c}(t)\right)\right\}+\sum_{s=1}^{M} \gamma_{r s} q_{s} \frac{1}{2} \sum_{i=1}^{N} e_{i}(t)^{T} P e_{i}(t) \\
& +q_{r}\left\{\sum_{k=1}^{n} p_{k} \varrho_{k} \tilde{e}^{k}(t)^{T}\left[A(r)-\Xi+\left(\breve{\delta}+\alpha_{r}\right) I_{N}\right] \tilde{e}^{k}(t)\right. \\
& \left.+\sum_{k=1}^{n} p_{k} \varrho_{k} \tilde{e}^{k}(t)^{T} B(r) \tilde{e}^{k}\left(t-\tau_{c}(t)\right)-\sum_{k=1}^{n} p_{k} \varrho_{k} \tilde{e}^{k}\left(t-\tau_{c}(t)\right)^{T} \beta_{r} \tilde{e}^{k}\left(t-\tau_{c}(t)\right)\right\} \\
& \leq q_{r}\left\{\sum_{i=1}^{N} e_{i}(t)^{T}\left(-\eta I_{N}+\frac{1}{2} \check{p} \sum_{j=1}^{N} \Upsilon_{j 1}^{r}-\alpha_{r} P \Sigma\right) e_{i}(t)+\sum_{i=1}^{N} e_{i}(t-\tau(t))^{T}\right.
\end{aligned}
$$




$$
\begin{aligned}
& \times\left(\beta_{r} I_{N}+\frac{1}{2} \check{p} \sum_{j=1}^{N} \Upsilon_{j 2}^{r}\right) e_{i}(t-\tau(t))+\sum_{i=1}^{N} e_{i}\left(t-\tau_{c}(t)\right)^{T}\left(\frac{1}{2} \breve{p} \sum_{j=1}^{N} \Upsilon_{j 3}^{r}+\beta_{r} P \Sigma\right) \\
& \left.\times e_{i}\left(t-\tau_{c}(t)\right)\right\}+\sum_{s=1}^{M} \gamma_{r s} q_{s} \frac{1}{2} \sum_{i=1}^{N} e_{i}(t)^{T} P e_{i}(t) .
\end{aligned}
$$

Let

$$
E(t)=\frac{1}{2} \sum_{i=1}^{N} e_{i}(t)^{T} P e_{i}(t)
$$

Then we have

$$
\mathcal{L} V(t) \leq-a_{r} q_{r} E(t)+b_{r} q_{r} E(t-\tau(t))+c_{r} q_{r} E\left(t-\tau_{c}(t)\right)+\sum_{s=1}^{M} \gamma_{r s} q_{s} E(t)
$$

and by (3.8), we have

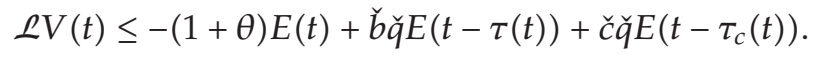

Define

$$
W(t)=e^{\gamma t} V(t)
$$

and use (3.13) to compute the operator

$$
\begin{aligned}
\mathcal{L} W(t) & =e^{\gamma t}[\gamma V(t)+\mathcal{L} V(t)] \\
& \leq e^{\gamma t}\left[\gamma \breve{p} E(t)-(1+\theta) E(t)+\breve{b} \breve{q} E(t-\tau(t))+\check{c} \breve{q} E\left(t-\tau_{c}(t)\right)\right],
\end{aligned}
$$

which, after applying the generalized Itô's formula, gives

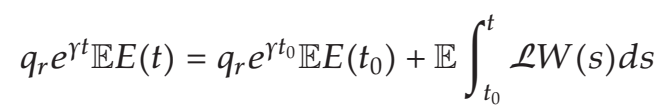


for any $t>t_{0} \geq 0$. Hence we have

$$
\begin{aligned}
q_{r} e^{\gamma t} \mathbb{E} E(t) \leq & \check{q} \mathbb{E} E(0)+\mathbb{E} \int_{0}^{t} e^{\gamma s}\left[\gamma E(s)-(1-\theta) E(s)+\check{b} \breve{q} E(s-\tau(s))+\check{c} \breve{q} E\left(s-\tau_{c}(s)\right)\right] d s \\
\leq & \frac{\check{p} \breve{q}}{2} \sum_{i=1}^{N} \mathbb{E}\left\|\xi_{i}\right\|^{2}+(\gamma-(1+\theta)) \int_{0}^{t} e^{\gamma s} \mathbb{E} E(s) d s+\breve{b} \breve{q} e^{\gamma \tau} \int_{0}^{t} e^{\gamma(s-\tau(s))} \mathbb{E} E(s-\tau(s)) d s \\
& +\check{c} \check{q} e^{\gamma \tau_{c}} \int_{0}^{t} e^{\gamma\left(s-\tau_{c}(s)\right)} \mathbb{E} E\left(s-\tau_{c}(s)\right) d s,
\end{aligned}
$$

which, by using the change of variables $s-\tau(s)=u$, gives

$$
\begin{aligned}
\int_{0}^{t} e^{\gamma(s-\tau(s))} \mathbb{E} E(s-\tau(s)) d s & =\int_{-\tau(0)}^{t-\tau(t)} e^{\gamma u} \mathbb{E} E(u) \frac{d u}{1-\dot{\tau}(t)} \\
& \leq \frac{\check{p} \tau}{2(1-\bar{\tau})} \sum_{i=1}^{N} \mathbb{E}\left\|\xi_{i}\right\|^{2}+\frac{1}{1-\bar{\tau}} \int_{0}^{t} e^{\gamma u} \mathbb{E} E(u) d u,
\end{aligned}
$$

and a further change of variables $s-\tau_{c}(s)=u$ gives

$$
\begin{aligned}
\int_{0}^{t} e^{s-\tau_{c}(s)} \mathbb{E} E\left(s-\tau_{c}(s)\right) d s & =\int_{-\tau_{c}(0)}^{t-\tau_{c}(t)} e^{\gamma u} \mathbb{E} E(u) \frac{d u}{1-\dot{\tau}_{c}(t)} \\
& \leq \frac{\check{p} \tau_{c}}{2\left(1-\bar{\tau}_{c}\right)} \sum_{i=1}^{N} \mathbb{E}\left\|\xi_{i}\right\|^{2}+\frac{1}{1-\bar{\tau}_{c}} \int_{0}^{t} e^{r u} \mathbb{E} E(u) d u .
\end{aligned}
$$

By Condition (3.4), we obtain

$$
\mathbb{E} E(t) \leq \frac{\check{p} \breve{q}}{2}\left(1+\frac{\breve{b} \breve{q} \tau}{1-\bar{\tau}} e^{\gamma \tau}+\frac{\check{c} \breve{q} \tau_{c}}{1-\bar{\tau}_{c}} e^{\gamma \tau_{c}}\right) \sum_{i=1}^{N} \mathbb{E}\left\|\xi_{i}\right\|^{2} e^{-\gamma t}
$$

so that

$$
\mathbb{E}\|e(t)\|^{2} \leq \frac{\check{p} \breve{q}}{2 \widehat{p}}\left(1+\frac{\check{b} \breve{q} \tau}{1-\bar{\tau}} e^{\gamma \tau}+\frac{\check{c} \breve{q} \tau_{c}}{1-\bar{\tau}_{c}} e^{\gamma \tau_{c}}\right) \sum_{i=1}^{N} \mathbb{E}\left\|\xi_{i}\right\|^{2} e^{-\gamma t}
$$

The proof is hence complete.

When the time-varying delays are constant (i.e., $\tau(t)=\tau, \tau_{c}(t)=\tau_{c}$ ), we obtain the following corollary. 
Corollary 3.2. Let assumptions ( $\mathrm{H} 1)$ and $(\mathrm{H} 2)$ be true and let $f \in \operatorname{QUAD}(P, \Delta, \eta, \theta)$. If there exist positive constants $\alpha_{r}$ and $\beta_{r}$ such that

$$
\begin{gathered}
{\left[\begin{array}{cc}
A(r)^{S}+\check{\delta} I_{N}-\Xi+\alpha_{r} I_{N} & \frac{B(r)}{2} \\
\frac{B(r)^{T}}{2} & -\beta_{r} I_{N}
\end{array}\right] \leq 0, \quad \text { for } r=1,2, \ldots, M,} \\
\check{\tau} \leq \theta T, \quad \check{\tau} \leq(1-\theta) T \\
\left(\frac{1}{b_{1}+c_{1}}, \frac{1}{b_{2}+c_{2}}, \ldots, \frac{1}{b_{M}+c_{M}}\right)>\widetilde{\Gamma}^{-1} \mathbf{1}_{M},
\end{gathered}
$$

where $r>0$ is the greatest root of the equation

$$
\begin{gathered}
\check{q} r-(1+\theta)+\check{b} \breve{q} e^{r \tau}+\check{c} \breve{q} e^{r \tau_{c}}=0, \\
\widetilde{\Gamma}=\operatorname{diag}\left\{a_{1}, a_{2}, \ldots, a_{M}\right\}-\Gamma, \\
a_{r}=\frac{\lambda_{\min }\left(2 \eta I_{n}-\check{p} \sum_{i=1}^{N} \Upsilon_{i 1}^{r}+2 \alpha_{1} P \Sigma\right)}{\check{p}}, \quad \check{a}=\max _{r \in S} a_{r}, \\
b_{r}=\frac{\lambda_{\max }\left(\sum_{i=1}^{N} P \Upsilon_{i 2}^{r}+2 \zeta I_{N}\right)}{\hat{p}}, \quad \check{b}=\max _{r \in S} b_{r}, \\
c_{r}=\frac{\lambda_{\max }\left(\sum_{i=1}^{N} P \Upsilon_{i 3}^{r}+2 \beta_{2} P \Sigma\right)}{\hat{p}}, \quad \check{c}=\max _{r \in S} c_{r},
\end{gathered}
$$

then the solutions $x_{1}(t), x_{1}(t), \ldots$ and $x_{N}(t)$ of system (2.9) are globally and exponentially stable.

When $A(r(t))=A, B(r(t))=B$, and $\sigma_{i}\left(t, e(t), e(t-\tau(t)), e\left(t-\tau_{c}(t)\right), r(t)\right)=\sigma_{i}(t, e(t)$, $\left.e(t-\tau(t)), e\left(t-\tau_{c}(t)\right)\right)$, we can get the following corollary.

Corollary 3.3. Let assumptions (H1) and (H2) be true, and let $f \in \operatorname{QUAD}(P, \Delta, \eta, \theta)$. If there exist positive constants $\alpha_{r}$ and $\beta_{r}$ such that

$$
\begin{gathered}
{\left[\begin{array}{cc}
A^{s}+\check{\delta} I_{N}-\Xi+\alpha_{r} I_{N} & \frac{B}{2} \\
\frac{B^{T}}{2} & -\beta_{r} I_{N}
\end{array}\right] \leq 0,} \\
\check{\tau} \leq \theta T, \quad \check{\tau} \leq(1-\theta) T, \quad 0 \leq \check{\tau} \leq 1-\frac{(\check{b}+\check{c})}{1+\theta},
\end{gathered}
$$


where

$$
\begin{gathered}
a_{r}=\frac{\lambda_{\min }\left(2 \eta I_{n}-\check{p} \sum_{i=1}^{N} \Upsilon_{i 1}^{r}+2 \alpha_{1} P \Sigma\right)}{\check{p}}, \quad \check{a}=\max _{r \in S} a_{r}, \\
b_{r}=\frac{\lambda_{\max }\left(\sum_{i=1}^{N} P \Upsilon_{i 2}^{r}+2 \zeta I_{N}\right)}{\hat{p}}, \quad \check{b}=\max _{r \in S} b_{r}, \\
c_{r}=\frac{\lambda_{\max }\left(\sum_{i=1}^{N} P \Upsilon_{i 3}^{r}+2 \beta_{2} P \Sigma\right)}{\hat{p}}, \quad \check{c}=\max _{r \in S} c_{r},
\end{gathered}
$$

then the solutions $x_{1}(t), x_{1}(t), \ldots$ and $x_{N}(t)$ of system (2.9) are globally and exponentially stable.

\section{Numerical Simulation}

In this section, we present some numerical simulation results that validate the theorem of the previous section.

Consider the chaotic delayed neural network

$$
d s(t)=[-C s(t)+A f(s(t))+B g(s(t-\tau(t)))] d t
$$

where $f(s)=g(s)=\tanh (s), \tau(t)=1$,

$$
C=\left[\begin{array}{ll}
1 & 0 \\
0 & 1
\end{array}\right], \quad A=\left[\begin{array}{cc}
2 & -0.1 \\
-5 & 4.5
\end{array}\right], \quad B=\left[\begin{array}{cc}
-1.5 & -0.1 \\
-0.2 & -4
\end{array}\right]
$$

Taking $P=\operatorname{diag}\{1,2\}$ and $\Delta=\operatorname{diag}\{5,11,5\}$, we have $\eta=0.15$ and $\theta=3.25$ so that Condition (2.11) is satisfied. Thus

$$
\begin{aligned}
d x_{i}(t)= & \left\{f\left(t, x_{i}(t), x_{i}(t-\tau(t))\right)+\sum_{j=1}^{5} a_{i j}^{r} \Sigma x_{j}(t)+\sum_{j=1}^{5} b_{i j}^{r} \Sigma x_{j}\left(t-\tau_{c}(t)\right)-\varepsilon_{i}\left(x_{i}(t)-s(t)\right)\right\} d t \\
& +\sigma_{i}^{r}\left(t, x(t), x(t-\tau(t)), x\left(t-\tau_{c}(t)\right)\right) d w_{i}(t), \quad i=1,2, \ldots, 5, r=1,2,3
\end{aligned}
$$




$$
\begin{aligned}
& A_{1}=\left[a_{i j}^{1}\right]=20\left[\begin{array}{ccccc}
-3 & 1 & 1 & 1 & 0 \\
1 & -4 & 1 & 1 & 1 \\
1 & 1 & -4 & 1 & 1 \\
1 & 1 & 1 & -4 & 1 \\
0 & 1 & 1 & 1 & -3
\end{array}\right], \quad A_{2}=\left[a_{i j}^{2}\right]=15\left[\begin{array}{ccccc}
-4 & 1 & 1 & 1 & 1 \\
1 & -3 & 1 & 1 & 0 \\
1 & 1 & -4 & 1 & 1 \\
1 & 1 & 1 & -4 & 1 \\
1 & 0 & 1 & 1 & -3
\end{array}\right] \text {, } \\
& A_{3}=\left[a_{i j}^{3}\right]=37\left[\begin{array}{ccccc}
-2 & 1 & 1 & 0 & 0 \\
1 & -3 & 1 & 1 & 0 \\
1 & 1 & -3 & 0 & 1 \\
0 & 1 & 0 & -1 & 0 \\
0 & 0 & 1 & 0 & -1
\end{array}\right], \quad B_{1}=\left[b_{i j}^{1}\right]=0.1\left[\begin{array}{ccccc}
-2 & 1 & 1 & 0 & 0 \\
0 & -2 & 1 & 1 & 0 \\
0 & 1 & -2 & 1 & 0 \\
0 & 1 & 1 & -2 & 0 \\
0 & 1 & 1 & 0 & -2
\end{array}\right] \\
& B_{2}=\left[b_{i j}^{2}\right]=0.2\left[\begin{array}{ccccc}
-1 & 0 & 0 & 1 & 0 \\
0 & -1 & 0 & 1 & 0 \\
0 & 1 & -1 & 0 & 0 \\
0 & 1 & 0 & -1 & 0 \\
0 & 0 & 0 & 1 & -1
\end{array}\right], \quad B_{3}=\left[b_{i j}^{3}\right]=0.7\left[\begin{array}{ccccc}
-1 & 0 & 1 & 0 & 0 \\
1 & -1 & 0 & 0 & 0 \\
0 & 0 & -1 & 1 & 0 \\
0 & 0 & 1 & -1 & 0 \\
1 & 0 & 0 & 0 & -1
\end{array}\right] \\
& \Xi=100\left[\begin{array}{lllll}
1 & 0 & 0 & 0 & 0 \\
0 & 1 & 0 & 0 & 0 \\
0 & 0 & 0 & 0 & 0 \\
0 & 0 & 0 & 0 & 0 \\
0 & 0 & 0 & 0 & 0
\end{array}\right], \quad \Gamma=\left[\begin{array}{ccc}
-3 & 1 & 2 \\
2 & -4 & 2 \\
2 & 3 & -5
\end{array}\right], \quad \tau_{c}(t)=0.1 \frac{e^{t}}{1+e^{t}} \\
& \sigma_{i}^{1}\left(t, x(t), x(t-\tau(t)), x\left(t-\tau_{c}(t)\right)\right)=0.1 \operatorname{diag}\left\{x_{i 1}(t)-x_{i+1,1}(t), x_{i 2}(t)-x_{i+1,2}(t)\right\}, \\
& \sigma_{i}^{2}\left(t, x(t), x(t-\tau(t)), x\left(t-\tau_{c}(t)\right)\right)=0.1 \operatorname{diag}\left\{x_{i 1}(t-\tau(t))-x_{i+1,1}(t-\tau(t)), x_{i 2}(t-\tau(t))\right. \\
& \left.-x_{i+1,2}(t-\tau(t))\right\}, \\
& \sigma_{i}^{3}(t, x(t), x(t-\tau(t)), x(t-\tau(t)))=0.1 \operatorname{diag}\left\{x_{i 1}\left(t-\tau_{c}(t)\right)-x_{i+1,1}\left(t-\tau_{c}(t)\right), x_{i 2}\left(t-\tau_{c}(t)\right)\right. \\
& \left.-x_{i+1,2}\left(t-\tau_{c}(t)\right)\right\} \text {. }
\end{aligned}
$$

Computations then yield $\tau=1, \bar{\tau}=0, \tau_{c}=0.1, \bar{\tau}_{c}=0.1, \Upsilon_{i j}=0.01 I_{2}$ for $i=1,2, \ldots, N$, and $j=1,2$. Let $J=\{1,2\}$ and the control strength $\varepsilon_{i}=100$ for $i=1,2$. Then the solutions of (3.1)-(3.3) are (by using the MATLAB LMI toolbox): $\alpha_{1}=3.5000, \beta_{1}=0.0020, a_{1}=7.1351$, $b_{1}=6.5300, c_{1}=0.0379 ; \alpha_{2}=3.6000, \beta_{2}=0.0088, a_{2}=7.3351, b_{2}=6.5300, c_{2}=0.0651$; $\alpha_{3}=3.7000, \beta_{3}=0.0852, a_{3}=7.5351, b_{3}=6.5300, c_{3}=0.3709$. So $\theta=0.003$, and

$$
\widetilde{\Gamma}^{-1}=\left[\begin{array}{ccc}
0.10526 & 0.014337 & 0.019082 \\
0.022486 & 0.095174 & 0.018773 \\
0.022176 & 0.025065 & 0.087313
\end{array}\right] .
$$

Therefore

$$
q=[0.1391,0.1368,0.1350]^{T},
$$

and, after solving (3.4), we obtain $\gamma=0.0349$. 


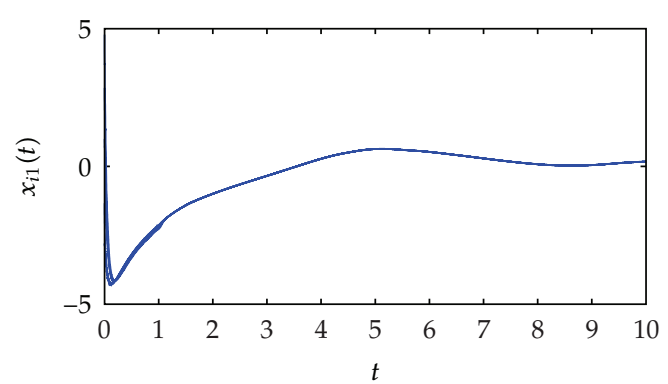

(a)

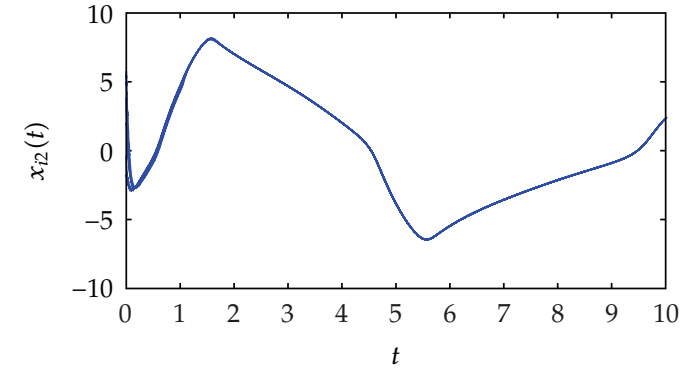

(b)

Figure 1: The trajectories of the state variables of $x_{i 1}$ and $x_{i 2}(i=1,2, \ldots, 5)$ in system (4.3) under pinning control.

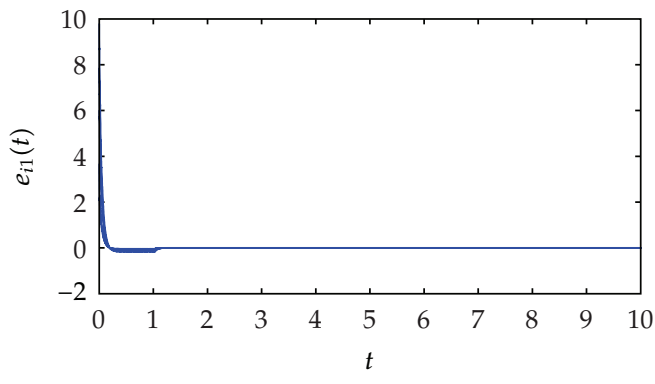

(a)

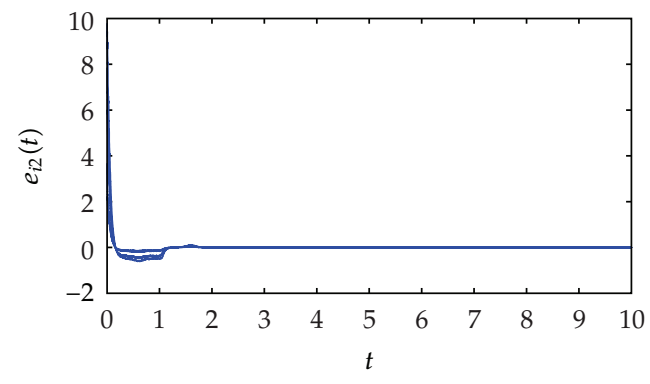

(b)

Figure 2: The time evolution of $e_{i 1}$ and $e_{i 2}(i=1,2, \ldots, 5)$ in system (4.3) under pinning control.

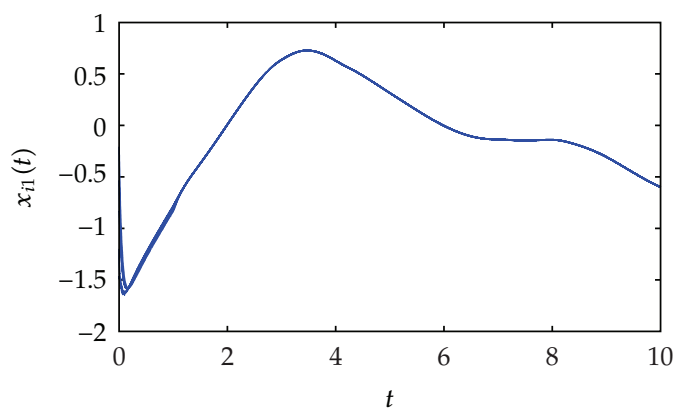

(a)

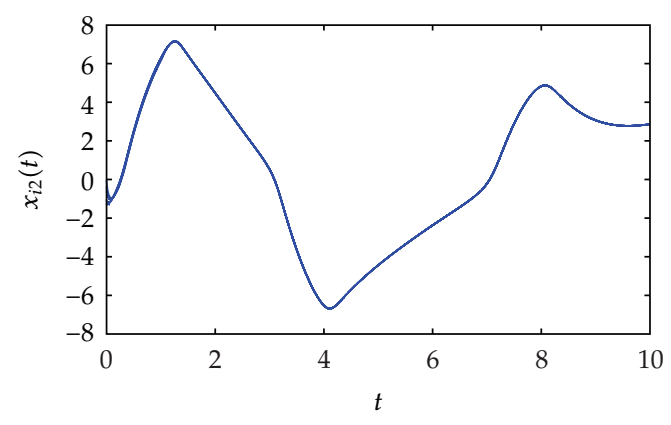

(b)

Figure 3: The trajectories of the state variables of $x_{i 1}$ and $x_{i 2}(i=1,2, \ldots, 5)$ in system (4.3) under pinning control.

The initial conditions for this simulation are $x_{i j}\left(t_{0}\right)=-6+2 i+j, i=1,2, \ldots, 5$, $j=1,2$ and $s\left(t_{0}\right)=[-5,-4]^{T}$ for all $t_{0} \in[-1,0]$ and the trajectories of the periodically intermittent pinning control gains are shown in Figure 1. Figure 2 shows the time evolution of the synchronization errors with periodically intermittent pinning control.

Next, let $t_{c}(t)=0.1$, we get the $\gamma=0.0402$. The initial conditions for this simulation are $x_{i j}\left(t_{0}\right)=-6+2 i+j, i=1,2, \ldots, 5, j=1,2$ and $s\left(t_{0}\right)=[-5,-4]^{T}$ for all $t_{0} \in[-1,0]$ and the trajectories of the periodically intermittent pinning control gains are shown in Figure 3. 


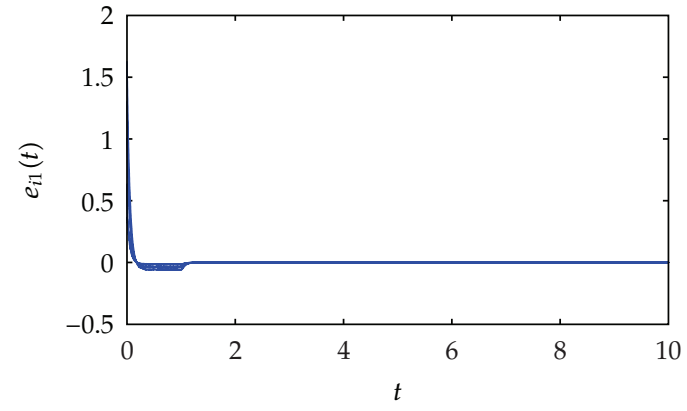

(a)

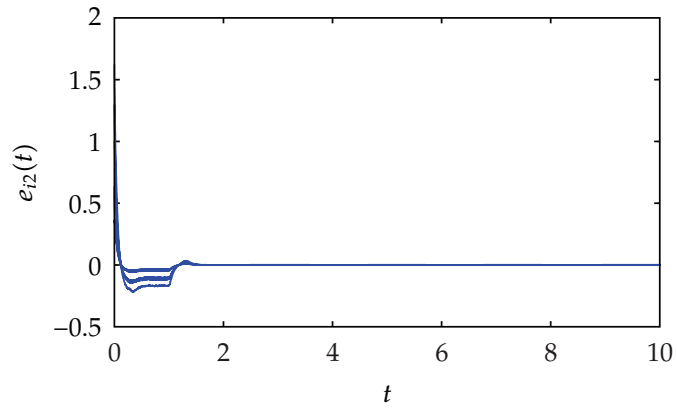

(b)

Figure 4: The time evolution of $e_{i 1}$ and $e_{i 2}(i=1,2, \ldots, 5)$ in system (4.3) under pinning control.

Figure 4 shows the time evolution of the synchronization errors with periodically intermittent pinning control.

\section{Conclusion}

In this paper, we investigated the synchronization problem for stochastic complex networks with Markovian switching and nondelayed and time-varying delayed couplings. Specifically, we achieved global exponential synchronization by applying a pinning control scheme to a small fraction of the nodes and derived sufficient conditions for global exponential stability of synchronization in mean square. Finally, we considered some numerical examples that illustrate the theoretical analysis.

\section{Acknowledgments}

This work was supported by the National Science Foundation of China under Grant no. 61070087, the Guangdong Education University Industry Cooperation Project (2009B090300355), and the Shenzhen Basic Research Project (JC201006010743A, JC200903120040A). The research of the authors is partially supported by the Hong Kong Polytechnic University Grant G-U996 and Hong Kong Government GRF Grant B-Q21F.

\section{References}

[1] A. Arenas, A. Díaz-Guilera, J. Kurths, Y. Moreno, and C. Zhou, "Synchronization in complex networks," Physics Reports A, vol. 469, no. 3, pp. 93-153, 2008.

[2] S. H. Strogatz and I. Stewart, "Coupled oscillators and biological synchronization," Scientific American, vol. 269, no. 6, pp. 102-109, 1993.

[3] C. M. Gray, "Synchronous oscillations in neuronal systems: mechanisms and functions," Journal of Computational Neuroscience, vol. 1, no. 1-2, pp. 11-38, 1994.

[4] M. S. De Vieira, "Chaos and synchronized chaos in an earthquake model," Physical Review Letters, vol. 82, no. 1, pp. 201-204, 1999.

[5] L. Kuhnert, K. I. Agladze, and V. I. Krinsky, "Image processing using light-sensitive chemical waves," Nature, vol. 337, no. 6204, pp. 244-247, 1989.

[6] D. Yu, M. Righero, and L. Kocarev, "Estimating topology of networks," Physical Review Letters, vol. 97, no. 18, Article ID 188701, 4 pages, 2006. 
[7] S. Boccaletti, J. Kurths, G. Osipov, D. L. Valladares, and C. S. Zhou, "The synchronization of chaotic systems," Physics Reports A, vol. 366, no. 1-2, pp. 1-101, 2002.

[8] R. Tönjes and B. Blasius, "Perturbation analysis of complete synchronization in networks of phase oscillators," Physical Review E, vol. 80, no. 2, Article ID 026202, 7 pages, 2009.

[9] V. N. Belykh, I. V. Belykh, and E. Mosekilde, "Cluster synchronization modes in an ensemble of coupled chaotic oscillators," Physical Review E, vol. 63, no. 3, pp. 362161-362164, 2001.

[10] M. G. Rosenblum, A. S. Pikovsky, and J. Kurths, "Phase synchronization of chaotic oscillators," Physical Review Letters, vol. 76, no. 11, pp. 1804-1807, 1996.

[11] C. Van Vreeswijk, "Partial synchronization in populations of pulse-coupled oscillators," Physical Review E, vol. 54, no. 5, pp. 5522-5537, 1996.

[12] X. Yang and J. Cao, "Adaptive pinning synchronization of complex networks with stochastic perturbations," Discrete Dynamics in Nature and Society, vol. 2010, Article ID 416182, 21 pages, 2010.

[13] G. P. Jiang, G. Chen, and W. K. S. Tang, "A new criterion for chaos synchronization using linear state feedback control," International Journal of Bifurcation and Chaos in Applied Sciences and Engineering, vol. 13, no. 8, pp. 2343-2351, 2003.

[14] M. Zochowski, “Intermittent dynamical control,” Physica D, vol. 145, no. 3-4, pp. 181-190, 2000.

[15] K. Tanaka and H. O. Wang, "Fuzzy control of chaotic systems using LMIs: regulation, synchronization and chaos model following," in Proceedings of the IEEE International Conference on Fuzzy Systems, pp. 434-439, May 1998.

[16] M. Haeri and M. Dehghani, "Impulsive synchronization of Chen's hyperchaotic system," Physics Letters, Section A, vol. 356, no. 3, pp. 226-230, 2006.

[17] T. Chen, X. Liu, and W. Lu, "Pinning complex networks by a single controller," IEEE Transactions on Circuits and Systems. I. Regular Papers, vol. 54, no. 6, pp. 1317-1326, 2007.

[18] J. Zhao, J. Lu, and X. Wu, "Pinning control of general complex dynamical networks with optimization," Science China. Information Sciences, vol. 53, no. 4, pp. 813-822, 2010.

[19] J. Zhou, J.-A. Lu, and J. Lü, "Pinning adaptive synchronization of a general complex dynamical network," Automatica, vol. 44, no. 4, pp. 996-1003, 2008.

[20] J. Zhao, J. A. Lu, and Q. Zhang, "Pinning a complex delayed dynamical network to a homogenous trajectory," IEEE Transactions on Circuits and Systems II, vol. 56, no. 6, pp. 514-518, 2009.

[21] C. Li and G. Chen, "Synchronization in general complex dynamical networks with coupling delays," Physica A, vol. 343, no. 1-4, pp. 263-278, 2004.

[22] W. Lu, T. Chen, and G. Chen, "Synchronization analysis of linearly coupled systems described by differential equations with a coupling delay," Physica D, vol. 221, no. 2, pp. 118-134, 2006.

[23] W. Wu and T. Chen, "Global synchronization criteria of linearly coupled neural network systems with time-varying coupling," IEEE Transactions on Neural Networks, vol. 19, no. 2, pp. 319-332, 2008.

[24] W. Guo, F. Austin, S. Chen, and W. Sun, "Pinning synchronization of the complex networks with non-delayed and delayed coupling," Physics Letters A, vol. 373, no. 17, pp. 1565-1572, 2009.

[25] S. Cai, Z. Liu, F. Xu, and J. Shen, "Periodically intermittent controlling complex dynamical networks with time-varying delays to a desired orbit," Physics Letters A, vol. 373, no. 42, pp. 3846-3854, 2009.

[26] S. Cai, J. Hao, Q. He, and Z. Liu, "Exponential synchronization of complex delayed dynamical networks via pinning periodically intermittent control," Physics Letters, Section A, vol. 375, no. 19, pp. 1965-1971, 2011.

[27] W. He and J. Cao, "Exponential synchronization of hybrid coupled networks with delayed coupling," IEEE Transactions on Neural Networks, vol. 21, no. 4, pp. 571-583, 2010.

[28] J. Cao, Z. Wang, and Y. Sun, "Synchronization in an array of linearly stochastically coupled networks with time delays," Physica A, vol. 385, no. 2, pp. 718-728, 2007.

[29] J. Liang, Z. Wang, Y. Liu, and X. Liu, "Global synchronization control of general delayed discretetime networks with stochastic coupling and disturbances," IEEE Transactions on Systems, Man, and Cybernetics, Part B, vol. 38, no. 4, pp. 1073-1083, 2008.

[30] A. Pototsky and N. Janson, "Synchronization of a large number of continuous one-dimensional stochastic elements with time-delayed mean-field coupling," Physica D, vol. 238, no. 2, pp. 175-183, 2009.

[31] X. Yang and J. Cao, "Stochastic synchronization of coupled neural networks with intermittent control," Physics Letters, Section A, vol. 373, no. 36, pp. 3259-3272, 2009.

[32] X. Mao, "Stability of stochastic differential equations with Markovian switching," Stochastic Processes and their Applications, vol. 79, no. 1, pp. 45-67, 1999.

[33] C. Yuan and X. Mao, "Robust stability and controllability of stochastic differential delay equations with Markovian switching," Automatica, vol. 40, no. 3, pp. 343-354, 2004. 
[34] Y. Sun and J. Cao, "Stabilization of stochastic delayed neural networks with Markovian switching," Asian Journal of Control, vol. 10, no. 3, pp. 327-340, 2008.

[35] Z. Wang, Y. Liu, L. Yu, and X. Liu, "Exponential stability of delayed recurrent neural networks with Markovian jumping parameters," Physics Letters, Section A, vol. 356, no. 4-5, pp. 346-352, 2006.

[36] G. Wang, J. Cao, and J. Liang, "Exponential stability in the mean square for stochastic neural networks with mixed time-delays and Markovian jumping parameters," Nonlinear Dynamics, vol. 57, no. 1-2, pp. 209-218, 2009.

[37] Y. Liu, Z. Wang, and X. Liu, "On synchronization of discrete-time markovian jumping stochastic complex networks with mode-dependent mixed time-delays," International Journal of Modern Physics B, vol. 23, no. 3, pp. 411-434, 2009. 


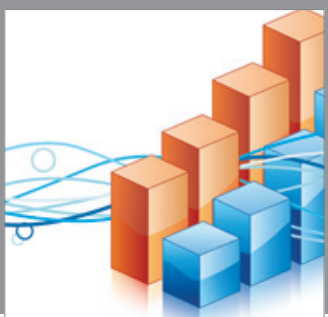

Advances in

Operations Research

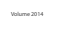

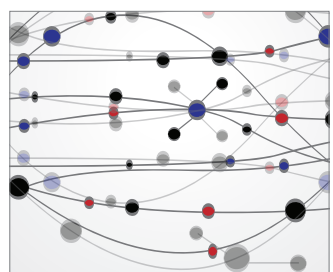

\section{The Scientific} World Journal
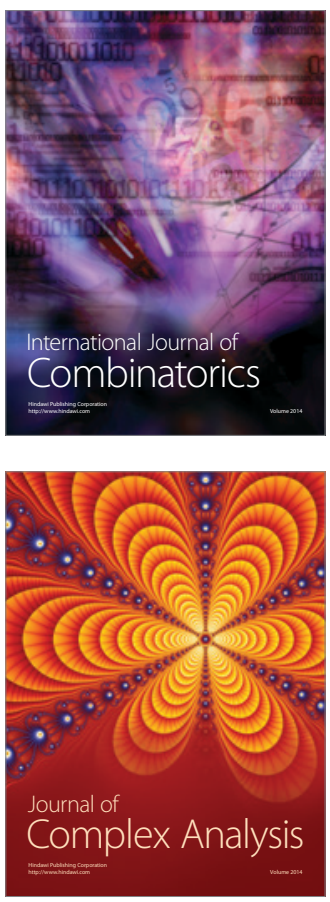

International Journal of

Mathematics and

Mathematical

Sciences
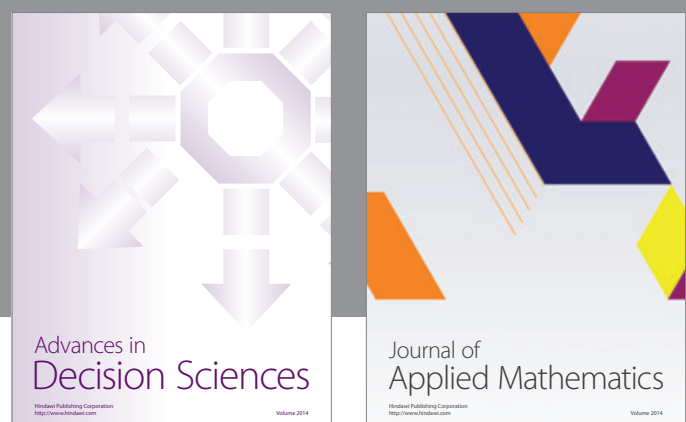

Journal of

Applied Mathematics
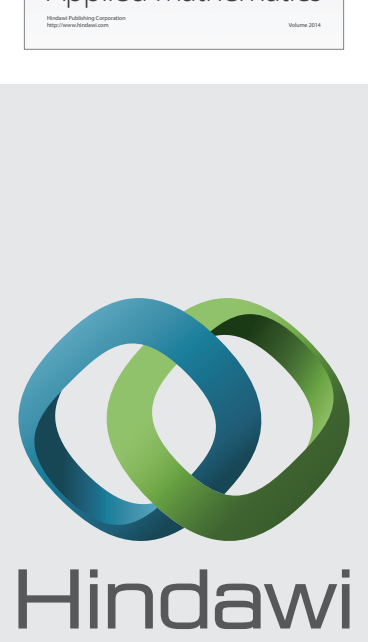

Submit your manuscripts at http://www.hindawi.com
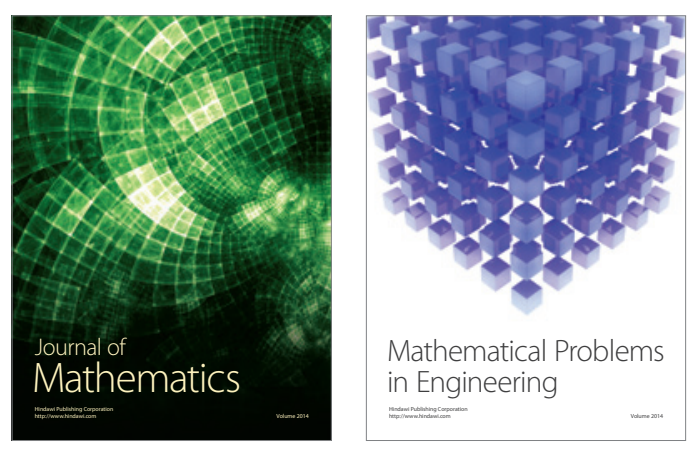

Mathematical Problems in Engineering
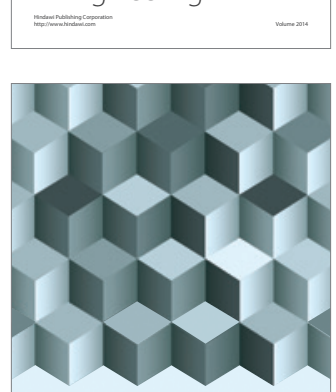

Journal of

Function Spaces
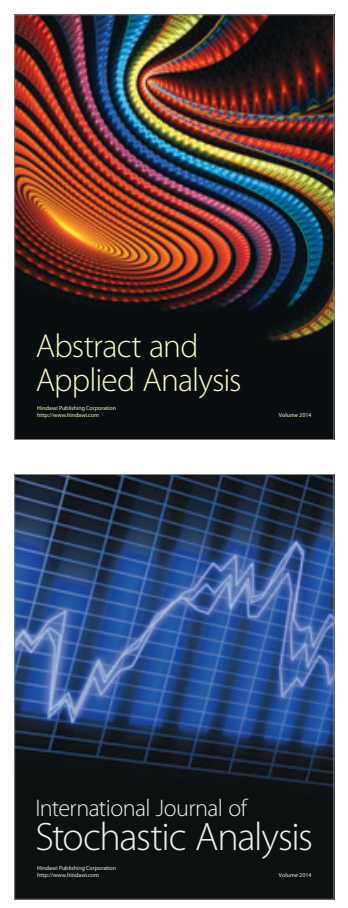

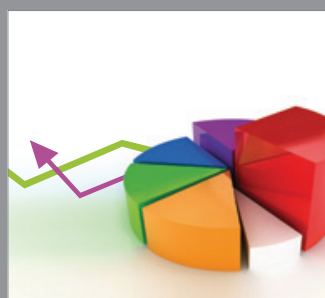

ournal of

Probability and Statistics

Promensencen
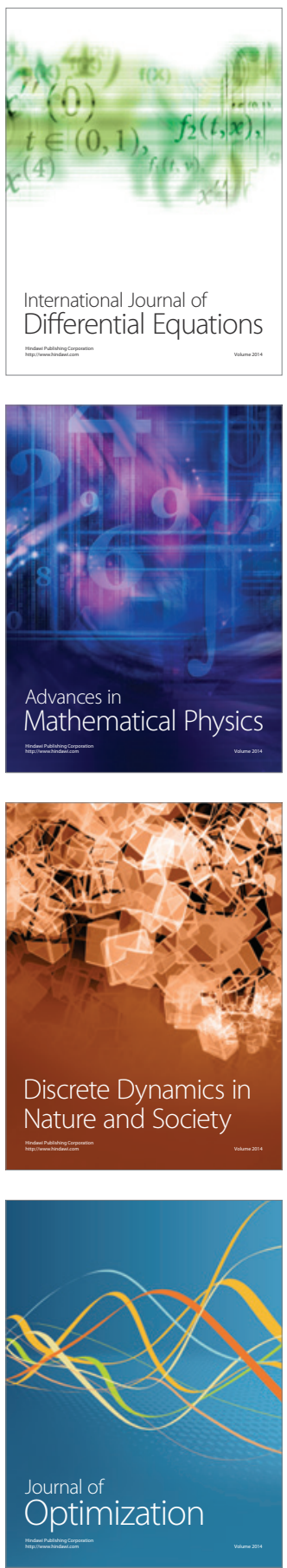